\title{
On Implementation Scheme of Agriculture Industrialization APP Based on Industrial 4
}

\author{
Sun Yanfei ${ }^{1, a}$,Wu Peng ${ }^{1, b}$ \\ ${ }^{1}$ ZIBO Vocational Institute, Shan Dong, 255000, P.R. China \\ ${ }^{\mathrm{a}}$ __sailing@sina.com, ${ }^{\mathrm{b}}$ email
}

Keywords: Industry 4; Agricultural industrialization; Cloud technology

\begin{abstract}
Intelligence based on the information physics system will enable humans to enter the fourth industrial revolution led by intelligent manufacturing in the next ten years, that is the industrial 4 era. Product of the whole life cycle and manufacturing process of digital and based on information communication technology template integration will form a highly flexible, personalized, digital products and services production model. According to the need of wisdom agriculture and achieve goal of agricultural industrialization , combined with mode of operation of agricultural production, this article analyses agricultural production related business processes and information systems processfrom services provided the supplier's perspective. This article highlights the business characteristics of Intelligent Agriculture in the planning of its management system, considering the actual demand of agriculture as well as the information transfer between the two sides of the supply and demand.
\end{abstract}

\section{Situation Analysis}

Industry 4.0. The Industry 4.0, it is based on intelligent manufacturing dominated the fourth industrial revolution. It is a new value model generated by the network, computer technology, information technology, software and automation technology depth interleaving with the high degree of integration of information technology and industrial technology. It will promote a new information physics system platform. The platform can connectall of the participation of persons, objects and systems. And it will provide comprehensive, efficient and secure services and applications to business processes. It also supports mobile devices and business networks of collaborative manufacturing, services, analysis and forecasting processes ,etc.

Current Status of the Research. Governments, social organizations, companies realize the big data brings the opportunities, and they promotetransboundary applications of big data in agriculture.

The Climate Corporation from California provides weather insurance project called Total Weather Insurance (TWI) for the agricultural growerscovering each season of the year.This projectprovides crop insurance to farmers by using specific data collection and analysis platform. it gets weather data form 2.5 million collection points everyday, and it can make an overall judgmentcombined with a lot of weather modeling, and plant roots and soil mass structure analysis on the risk of unexpected weather.

Japan Miyazaki southwestern “capital city” use cloud and big data foragricultural production. It collects the indicators of agricultural products through the sensor, camera and other kinds of terminals and applications, and it put the data together to the cloud for real-time monitoring, analysis and management.

In Britain, most of the farms have been to say goodbye to the hand milking.Automatic milking equipment penetration rate reached more than $90 \%$.Robots are not only milking, but also can be detected in the milking process. At present, most British cattle and pig and fish farms have achieved different levels of intelligence and automation management from the feed preparation, distribution, feeding to excrement cleaning. 


\section{Feasibility Analysis}

At present, productivity has been greatly promoted because agriculture has been completed by artificial, to semi-mechanized agriculture, to large-scale mechanization of agriculture. However, with the increasing pressure of population, the arable land can be reduced, the agriculture needs another change to meet the needs of human food production. The traditional mode of agricultural production should change to the data driven intelligent mode of production, and cloud computing, big data and the Internet and other science and technology will be the main driving force of this change.

Our government attaches great importance to the development of agriculture in our country.With the continuous development of the Internet of things technology, more and more technology is also applied to agricultural production.At present intelligent agriculture has achieved the monitoring of the various indicators of agricultural greenhouses, and it promotes the development of agricultural industry. However, these platforms transmitto the farmer's information in a single, fragmented and so on, and they can not provide a strong guidance to farmers.So the role of intelligent agriculture will be greatly reduced. Therefore, a rapid configuration, rapid implementation, rapid application, rapid and effective agricultural information technology application platform will be very obvious advantages.

\section{Scheme Design}

Guiding Ideology. From the need of intelligent agriculture and the goal of agricultural industrialization, combined with mode of operation of agricultural production, it analysisagricultural production related business processes and information systems processthink about it for the sake of the service provider's point of view. At the same time it takes into account the actual demand of agriculture and information transfer between the two sides of the supply and demand in the planning of its management system , and it highlights the business characteristics of Intelligent

Agriculture .

Scheme Architecture. The main function modules of the system are shown below:

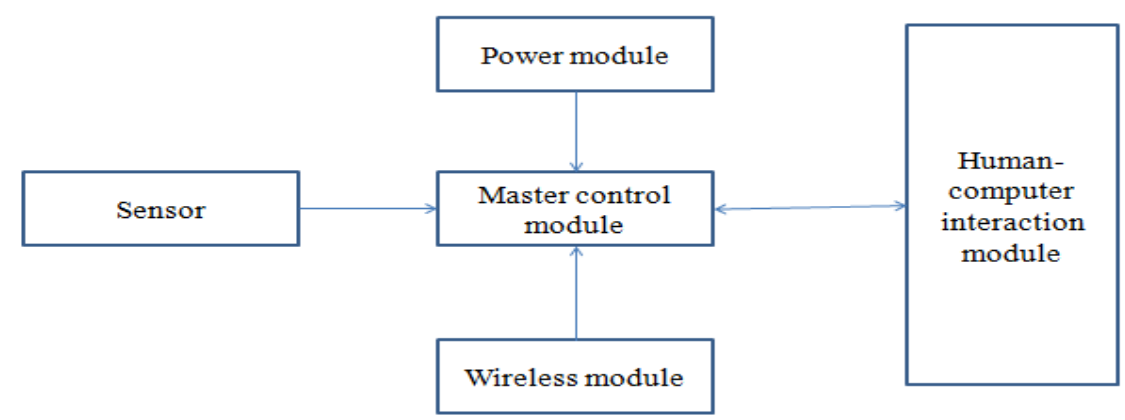

Figure 1System function module

We use object-oriented analysis, design and program to develop the software On the basis of architecture analysis and design.

We define software development in terms of the following aspects:thesoftware hardware and network environment;the subsystem and interface; the High level processes, protocols and interfaces; the data management rules; the design of connection, connection management, storage and so on.

We use the android application framework in the part of mobile terminal system application. The human-computer interaction model is established based on Activity, and the interface presentation, 
request initiation and response, content interaction and message content processing are established. By using the asynchronous HTTP, the interface blocking problem caused by the network request is solved. It realizes the local database and server database synchronization to achieve the purpose of saving flow, smooth interface combined with the Android data storage mode.

We use relative simple design style to the interface designconsidering the characteristics of the farmers themselves.It can reduce the user to date text input, user-friendly operation.Data processing module uses XML analysis and JSON analysis method. It uses JSON way to complete the analysis for the analysis of the data less commonly, and it uses XML way to complete the analysis for large-scale data.In order to solve the problem of the interface blocking caused by the network request, the asynchronous HTTP mode is selected. And it uses Android's own database to realize the synchronization between the local database and the server database in order to achieve the purpose of saving flow and smooth interface. The project is further improved according to the project to complete the progress of it, including the maintenance of data, as well as the improvement of the function of WEB and so on.

Related implementation functions are as follows:

1) Simulation of various sensors and intelligent gateways in agricultural greenhouse, users can develop APP to read the data;

2) Processing all kinds of production data, feedback and control of the sensor action;

3) Set the normal range of various agricultural indicators through APP ,such as air temperature and humidity, soil temperature and humidity, carbon dioxide concentration, light intensity and so on;

4) Open the corresponding control equipment using APP,such as alarm, water spray, LED, etc., so as to achieve the effective management of intelligent agricultural greenhouse;

5) Achieve the main production line function, to help users to grasp the entire production process, control costs;

6) Achieve the real-time monitoring function, real-time display and agricultural production related to the production of various indicators;

7) Achieve agricultural discussion, system settings and other functions, the use of good interface design features for the platform to win more users;

8) Achieve the information symmetry between the two sides of supply and demand, to solve the problem of information asymmetry;

9) Solve the problem of the interface blocking caused by the network request by selecting the asynchronous HTTP.Achieve the local database and server database synchronization combined with the Android data storage mode.

Organizational Guarantee. It should encourage the participation of many parties to strengthen the agricultural development strategy and policy research. Encourage the participation of many parties to strengthen the agricultural development strategy and policy research. Carrying out agricultural IOT policy at the national level development work, strengthening government macro guidance on agricultural IOT work,encouraging all sectors of society to participate in the research, development, transformation, promotion and application of Agricultural Internet of things technology,and it put the application of support and development of agricultural IOT technology into the countrypolicy ofStrong agricultural Huinong, and Vernon.

It should increase the investment of agricultural science and technology in all walks of life. As the agricultural technology,agricultural IOT technology has the characteristic of weak foundation, overall investment strength. It benefits from the wide level, and social welfare is strong. At present, the efficiency of agricultural output is not high, the level of farmers' income is low, and the 
operation of agricultural information market is not perfect. The central and local governments should apply the policy and the impact of the two major advantages to increase its investment, overall planning and long-term planning. Development of agricultural IOT Technology should have priority consideration. It shouldencourage social capital power and capital power take part in development and construction work of agricultural IOT technology.

\section{Acknowledgements}

Zibo science and technology development project(No. 2015kj010047)

\section{Conclusion}

With the rapid development of industry 4, mobile Internet and big data, it is very important for making use of intelligent mobile phone real-time access to agricultural monitoring data and the guidance of the development of intelligent agriculture.This article hopes to make up for the deficiency of the existing market products, at the same time, it is close to the actual needs of the agricultural industry, and itcan effectively promote the process of the intelligent agricultural development and promote the rapid development of the agricultural industry management, production, transportation, processing.

\section{Reference}

[1] Yuan Xiaomin.Industrial structure upgrading in the era of big data[J]. Market Modernization.2015,(3).

[2] Huang Chaoqiong, Wang Dabao, Chen Chao,Zhang Li.Design and implementation of intelligent agriculture APP based on Android[J]. Software Guide,2015(1).

[3] Zhang Shujuan.Design and implementation of enterprise information management system based on BPM[J].Journal of Hubei University of Science and Technology.2014,34(3).

[4] Yang Darong.Development strategy of China's intelligent agricultural industry[J].Jiangsu Agricultural Sciences,2014(4).

[5] Yan Zhangpeng,Peng Cheng.On implementation scheme of the Internet of things technology in wisdom agriculture[J].Journal of Xi'an University of Posts and Telecommunications,2013,18(4).

[6] Xu Haibin,Wang Hongxiang,Yang Xiaolin,Cao Jiwen.Current situation and Prospect of the application of Internet of things in modern agriculture[J]. Jiangsu Agricultural Sciences. 2013(05).

[7] Ma Guojun.The core technology of Internet of things and its application in agriculture[J]. Jiangsu Agricultural Sciences. 2012(11).

[8] Zhu Hongbo,Yang Longxiang,Zhu Qi. Survey on the Internet of Things[J]. Journal of Nanjing University of Posts and Telecommunications(Natural Science Edition). 2011(01). 